\title{
Steroid Induced Perforation of Meckel's Diverticulum in an Adult
}

Palanisamy Senthilnathan ${ }^{1}$, Srivatsan Gurumurthy Sivakumar ${ }^{1}$, Sumanta Dey ${ }^{1}$, Senthil Anand Elavarasan ${ }^{1}$, Anand Vijai Natesan ${ }^{1}$, Aravinth Subramanium², Nalankilli Vaiyapuri Gounder Palanisamy ${ }^{1}$, Chinnusamy Palanivelu ${ }^{1}$

Departments of ${ }^{1}$ Surgical Gastroenterology and ${ }^{2}$ Pathology, GEM Hospital \& Research Centre, Coimbatore, Tamil Nadu, India.

\section{Corresponding Author:}

Dr. Sumanta Dey

Email: doc.sumanta@gmail.com

This is an Open Access article distributed under the terms of the Creative Commons Attribution License (creativecommons.org/ licenses/by/3.0).

Published

March 10, 2017

\begin{abstract}
Background: Perforation is one of the rare complications of Meckel's diverticulum (MD) which carries high morbidity and mortality. Being a congenital variant, complicated MD is usually seen in pediatric population. Case Report: An adult male diagnosed with psoriasis and on systemic steroids for last three months presented with generalized abdominal pain, and distension. Free fluid and gas in abdomen were detected in erect abdominal X-ray and CT scan. Exploratory laparotomy showed perforated MD. Histopathology report showed presence of ulceration and perforation near ectopic gastric mucosa. The normal diverticular mucosa was ulcerated and perforation was due to persistent low $\mathrm{pH}$ inside the diverticulum. Conclusion: High dose systemic steroid therapy may cause spontaneous MD perforation. Detailed history coupled with high degree of clinical and radiological suspicion is required to clinch the pre-operative diagnosis.
\end{abstract}

Keywords: Abdominal Pain, Laparotomy, Meckel Diverticulum, Psoriasis, X-Rays.

\section{Introduction}

The worldwide incidence of Meckel's diverticulum in general population is approximately $2 \%$. It may present with various complications but the incidence is even rarer. Spontaneous perforation of Meckel's diverticulum is one of the dreaded and rare complication with high mortality. We hereby report a steroid induced perforation of Meckel's diverticulum and its surgical management in adult male.

\section{Case Report}

A thirty three year old gentleman presented to our casualty with pain abdomen and abdominal distension for three days. This was accompanied by low grade fever and constipation. He never had similar problems in past. He was diagnosed with psoriasis and was on methyl prednisolone $20 \mathrm{mg}$ per day for last three months. He had no other associated complaints, co-morbid conditions, addictions or significant family history.

General physical examination showed conscious, alert, co-operative male having mild pallor and fever of $101^{\circ} \mathrm{F}$. He was having exfoliative erythematous skin lesions over most of his body parts which was diagnosed as psoriasis previously [Fig.1,2]. His pulse was 106/min, blood pressure 100/60 $\mathrm{mm} \mathrm{Hg}$, respiratory rate 18/min. Abdominal examination revealed generalized distension generalized tenderness along with rebound tenderness. Liver dullness was obliterated and free fluid was present.

After initial resuscitation, blood investigations were sent and imaging of abdomen were done. His blood reports showed leucocytosis. Erect X-ray abdomen [Fig.3] showed free gas under both domes of diaphragm and dilated small bowel loops. Contrast CT scan abdomen [Fig.4,5] 
was suggestive of distal ileal perforation. Thus, our provisional diagnosis was perforative peritonitis due to distal ileal perforation and patient was taken for emergency laparoscopy followed by exploratory laparotomy.

Under general anaesthesia, initial diagnostic laparoscopy revealed flakes all over small bowel and a probable Meckel's diverticular perforation. Abdominal exploration showed Meckel's diverticulum with solitary perforation [Fig.6] near base and small bowel loops were dilated with flakes all around. Approximately $10 \mathrm{~cm}$ of ileal segment containing Meckel's diverticulum was resected [Fig.7] and end-to-end ileo-ileal hand sewn anastomosis was done. The appendix was also removed to prevent future complications. After giving thorough peritoneal lavage abdomen was closed after keeping one drain tube in pelvis. The whole procedure was uneventful. He recovered well in post-operative period without any complications. He was discharged in a stable condition on day five. Before discharge dermatology opinion was taken and methotrexate tablet was started for psoriasis. The histopathology report was showing gastric metaplasia [Fig.8,9] and no evidence of dysplasia in the Meckel's diverticulum and the appendix showed sub-mucosal inflammation. The patient was on regular follow up at our outpatient department and he is doing well.

\section{Discussion}

Meckel's diverticulum was first described in 1809 in an autopsy series by Johann Meckel, the German anatomist/embryologist [1]. Symptomatic presentation of Meckel's diverticula is mostly seen in male pediatric population and include gastrointestinal haemorrhage, diverticulitis, obstruction, and perforation $[3,4]$. Perforation of Meckel's diverticulum is one of the uncommon complications with high morbidity and mortality, almost always preceded by diverticulitis [1-3]. The perforations can be NSAID induced [5], fish or

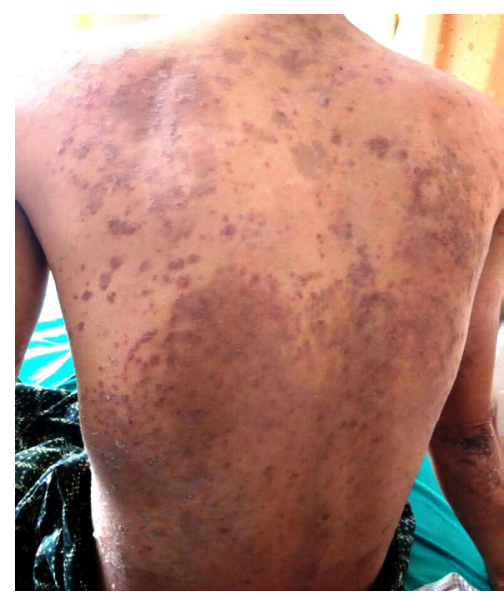

Fig.1: Psoriatic skin lesions over the back of the patient.

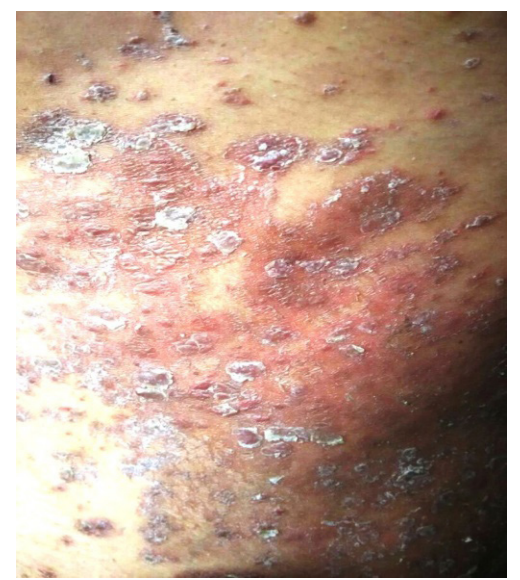

Fig.2: Exfoliating erythematous skin lesions characteristic of psoriasis.

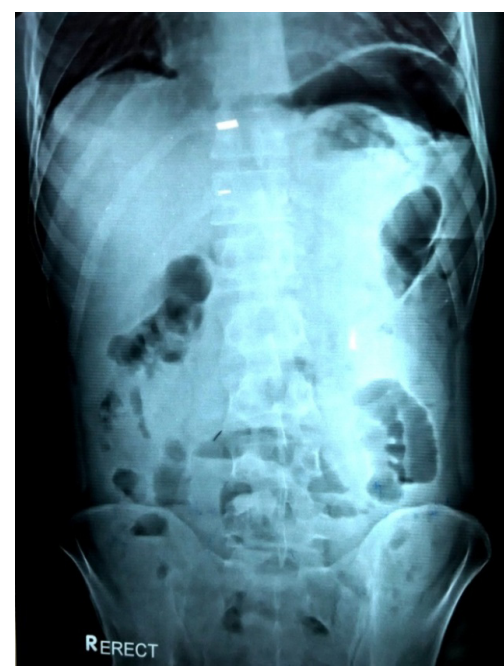

Fig.3: Straight X-ray abdomen in erect posture showing free gas under both dome of diaphragm with dilated bowel loops. 


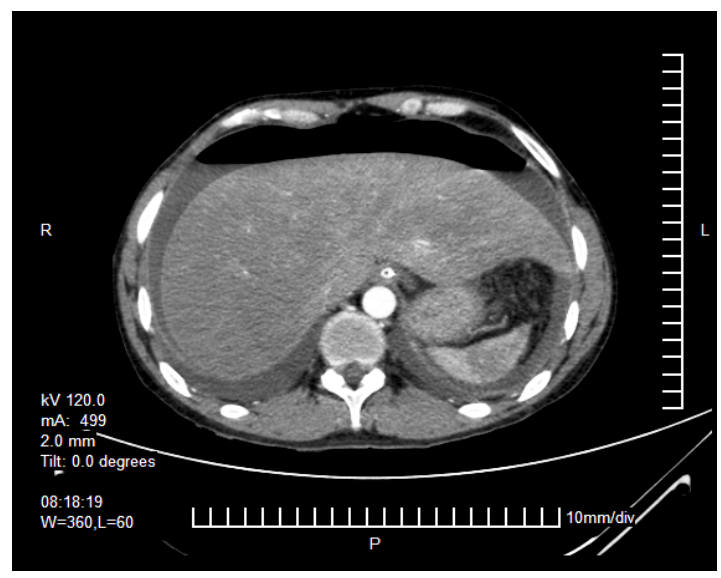

Fig.4: CECT abdomen showing free fluid gas inside peritoneal cavity suggestive of hollow viscus perforation.

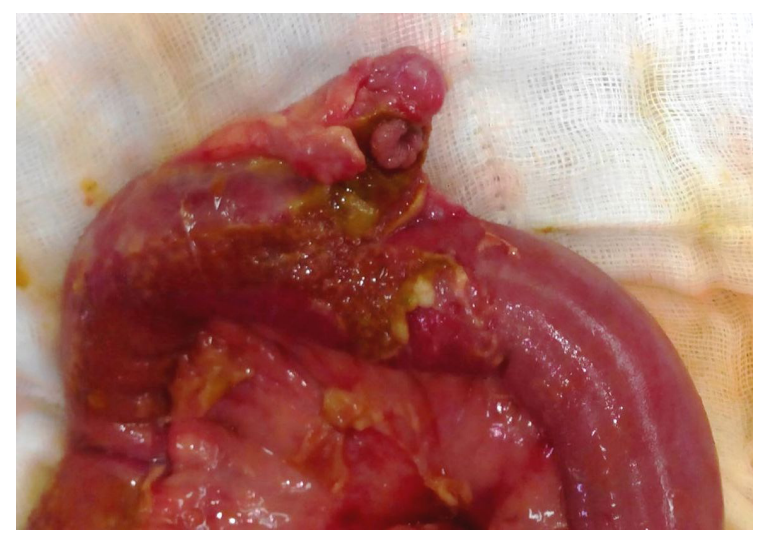

Fig.6: Intra-operative photograph showing Meckel's diverticulum (antimesentric border of terminal ileum) and solitary perforation.

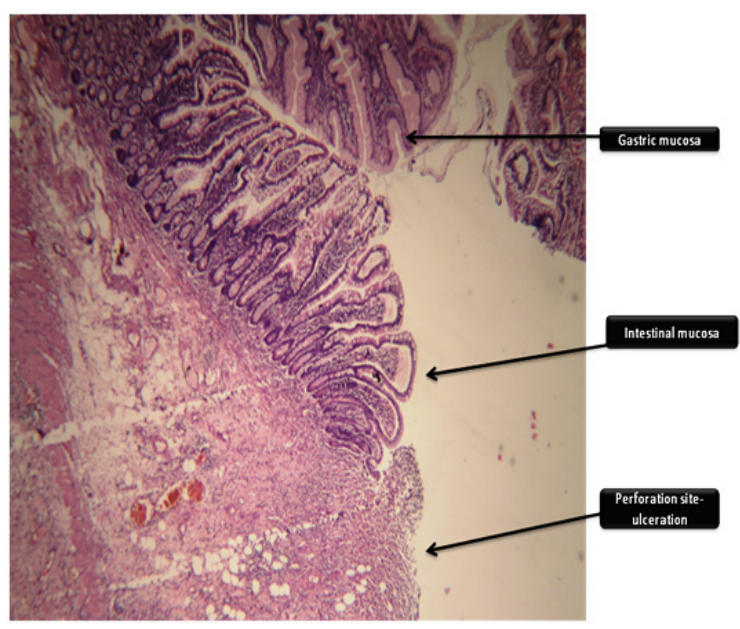

Fig.8: Histopathology slide showing perforation is adjacent to ectopic gastric mucosa.

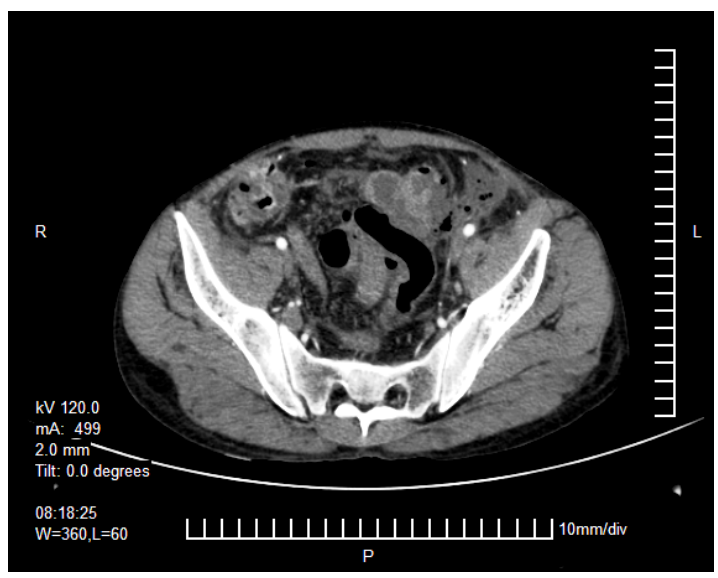

Fig.5: CECT abdomen showing air pockets, free fluids, dilated small bowel loops.

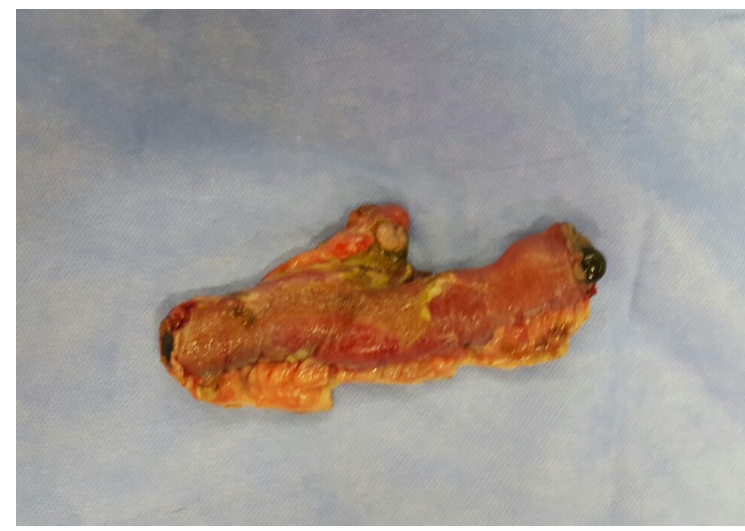

Fig.7: Resected segment of ileum containing perforated Meckel's diverticulum.

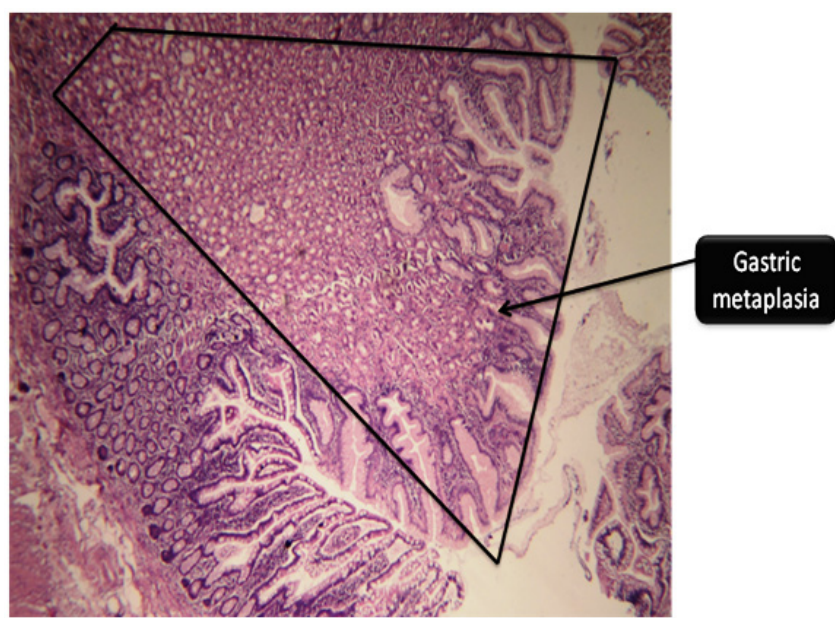

Fig.9: Histopathology slide showing gastric within Meckel's divertiulum. 
chicken bone impaction [6,7]. Steroid induced peptic perforation is a documented complications of long standing systemic steroid therapy [8,9]. Our patient was on oral prednisolone therapy for psoriasis 20 mg per day for last four months. This long duration steroid treatment stimulated the ectopic gastric mucosa in MD leading to excess acid production. The excess acid caused ulceration of adjacent intestinal mucosa which cannot tolerate low $\mathrm{pH}$ fluid. Ongoing ulceration process led to perforation of MD. Based on these evidences we can say that in our patient the etiology of perforation was long standing prednisolone therapy. However, the role of steroid treatment in psoriasis is doubtful [10]. There was no history suggestive of diverticulitis or diverticular bleeding before coming to our hospital. But, presence of mucosal ulceration and site of perforation in diverticular mucosa is well demonstrated by histopathology slides. The long standing prednisolone therapy might have masked the clinical features of ongoing diverticulitis [11]. Thus long standing steroid therapy induced the perforation without producing the typical clinical features of diverticulitis $[11,12]$.

Meckel's diverticulum and its complications especially perforation can be an elusive diagnosis, particularly in the adult patient [8]. Very few cases of perforated Meckel's diverticula in adults are diagnosed preoperatively by standard abdominal imaging studies such as CT scan and ultrasonography because of presence of air pockets, free fluids and bowel wall edema. Despite the previously mentioned modalities, most complicated Meckel's diverticula are still identified at the time of abdominal exploration. The technetium-99m pertechnate scan, or Meckel's scan, is generally regarded as the most accurate, non-invasive diagnostic technique $[4,8]$. However in emergency situations when a diagnosis of perforative peritonitis is well established one should go for diagnostic laparoscopy or exploratory laparotomy $[12,13]$. Our patient presented with features of diffuse peritonitis, dilated small bowel loops. Exploratory laparotomy identified MD perforation, histopathology revealed presence of ectopic gastric mucosa which had produced ulceration and perforation of adjacent intestinal mucosa of MD [Fig.8,9].

\section{Conclusion}

Perforation of MD itself is an uncommon complication and steroid induced perforation is even less documented. Detailed patient's history, experienced clinician and expert radiological opinion are required for pre-operative diagnosis.

Contributors: All authors have contributed equally in case management, review of literature, manuscript preparation and correction. SD will act as guarantor. All authors approved the final version of the manuscript.

Funding: None; Competing interests: None stated.

\section{References}

1. Kloss BT, Broton CE, Sullivan AM. Perforated Meckel diverticulum.Int J Emerg Med. 2010;3:455-457.

2. Sharma RK, Jain VK. Emergency surgery for Meckel's diverticulum. World J Emerg Surg. 2008;3:27.

3. McKay R. High incidence of symptomatic Meckel's diverticulum in patients less than fifty years of age: an indication for resection.. Am Surg. 2007;73(3):271-275.

4. Dumper J, Mackenzie S, Mitchell P, Sutherland F, Quan ML. Complications of Meckel's diverticula in adults. Can J Surg. 2006;49:353-357.

5. Brar AS, Gill RS, Gill SS, Wang H. NSAID-associated perforation of a Meckel's diverticulum : A case report, J Clin Med Res. 2011;3:96-98.

6. Wong JH, Suhaili DN, Kok KY. Fish bone perforation of Meckel's diverticulum: a rare event? Asian J Surg. 2005;28:295-296.

7. Yagci G, Cetinar S, Tufan T. Perforation of Meckel's diverticulumby a chiken bone, a rare complication : report of a case. Surg Today. 2004;34:606-608.

8. Sager J, Kumar V, Shah DK. Meckel's diverticulum: a systemic review. J R Soc Med. 2006;99:501-505.

9. Fraser JD, St Peter SD, Ostlie DJ. Perforated Meckel's diverticulum in a micro premature infant and review of the literature. Pediatr Surg Int. 2009;25:539-541.

10. Mrowietz U, Domm S. Systemic steroids in the treatment of psoriasis: what is fact, what is fiction? J Eur Acad Dermatol Venereol. 2013;27:1022-1025. 
11. Robert A, Nezamis JE. Effect of Prednisolone on Gastric Mucus Content and on Ulcer Formation. Experimental Biology and Medicine. 1963;114:545-550.

12. Liaw CC, Huang JS, Wang HM, Wang CH. Spontaneous gastroduodenal perforation in patients with cancer receiving chemotherapy and steroids. Report of four cases combining 5-fluorouracil infusion and cisplatin with antiemetics dexamethasone. Cancer. 1993;72:13821385.

13. ReMine SG, McIlrath DC. Bowel perforation in steroidtreated patients. Ann Surg. 1980;192:581-586. 